\title{
Endoscopia digestiva en tiempos de COVID-19: Resumen de recomendaciones
}

\author{
Digestive endoscopy in an era of COVID-19
}

\author{
Pilar Adriana Torres Mesa \\ Médica, Especialista en Cirugía General, Oncología Peritoneal, Cirugía Gastrointestinal y Endoscopia Digestiva, \\ Hospital Regional de Sogamoso y Centro Médico Especializado Gastrolife SAS, Duitama, Colombia.
}

\section{Introducción}

La situación actual de pandemia por COVID-19 ha puesto en evidencia las debilidades y necesidades no atendidas del sector salud en Colombia. Salieron a la luz las inequidades de un sistema económico y social, que desde hace varios años venía disminuyendo la protección laboral, económica y jurídica del talento humano en salud del país, que en estos momentos se ve enfrentando una batalla difícil, ante un enemigo todavía poco conocido.

$\mathrm{El} 7$ de enero de 2020, las autoridades chinas identificaron como agente causante del brote de neumonía de origen desconocido (informado el 3I de diciembre de 20I9), a un nuevo tipo de virus de la familia Coronaviridae, que fue denominado "nuevo coronavirus", SARS-COV-2 ${ }^{1,2}$. El
II de febrero de 2020, la Organización Mundial de la Salud (OMS), denomina a la enfermedad por infección por el Coronavirus del Síndrome Respiratorio Agudo Severo o Grave 2 (Severe respiratory acute syndrome 2), como Coronavirus Disease 2019 o COVID-1938.

La propagación de persona a persona se confirma, incluso entre los trabajadores de la salud que atienden personas con enfermedad por coronavirus (COVID-I9), lo cual es consistente con lo conocido sobre otros patógenos similares ${ }^{8,9}$. El II de marzo de 2020, la Organización Mundial de la Salud (OMS), declaró al brote de COVID-I9 como Pandemia, y emitió una serie de recomendaciones para que los países activen y amplíen sus mecanismos de respuesta a emergencias ${ }^{8, \mathrm{IO}}$.

Palabras clave: COVID-I9; virus del SRAS; coronavirus; infecciones por coronavirus; pandemias; endoscopía; endoscopía gastrointestinal.

Keywords: COVID-I9; SARS virus; coronavirus; coronavirus infections; pandemics; endoscopy; endoscopy, gastrointestinal.

Fecha de recibido: 04/04/2020 - Fecha de aceptación: 06/04/2020

Correspondencia: Pilar A. Torres M. Centro Médico Especializado Gastrolife SAS, Transversal 29 \# 9-78 Local 201, Duitama, Boyacá, Colombia, Celular: 3124317583

Correo electrónico: piadriana78@hotmail.com

Citar como: Torres Mesa PA. Endoscopia digestiva en tiempos de covid-19:

Resumen de recomendaciones. Rev Colomb Cir. 2020;35:209-15/Especial COVID-19. https://doi.org/10.30944/20117582.622

Este es un artículo de acceso abierto bajo una Licencia Creative Commons - BY-NC-ND https://creativecommons.org/licenses/by-ncnd/4.0/deed.es 
En Colombia, el 3I de marzo de 2020 el Ministerio de Salud y Protección Social, declara la fase de mitigación de COVID-I9 ${ }^{8,11}$.

\section{Entendiendo el coronavirus}

Los Coronavirus (CoV) son virus ARN de sentido positivo, encapsulados y no segmentados, de varios tipos (alfa, beta, gamma y delta), que generalmente causan enfermedades entéricas y respiratorias en animales y humanos. Los virus SARS-CoV y SARS-CoV-2 se unen a sus células objetivo a través de los receptores de la enzima convertidora de angiotensina 2 (ACE2), los cuales se expresan en células epiteliales de las vías respiratorias, parénquima pulmonar, endotelio vascular, células renales e intestino delgado ${ }^{\mathrm{I2}, 13}$.

\section{¿Cómo se apoderan los coronavirus de un} huésped?

El coronavirus es un virus que usa su ARN como plantilla directa para crear proteínas y un nuevo genoma en el citoplasma. Una vez el virus ingresa a la célula humana, toma el control de toda la célula. La mayoría de las organelas citoplasmáticas son engañadas y comienzan a trabajar para el virus. El virus ARN no puede producir más virus, por lo que obliga a la célula a producir grupos de proteínas, que se almacenan, ensamblan, encapsulan y transportan dentro de la célula. Finalmente, la progenie viral es transportada por las vesículas de Golgi hacia la membrana celular, donde son exocitadas hacia el espacio extracelular ${ }^{14,15}$.

El coronavirus es una nano partícula autoensamblada, que tiene como eslabón débil de su coraza, una capa lipídica. Por esta razón, lavarse las manos con agua y jabón, es la estrategia mas efectiva para eliminar el virus de la piel ${ }^{14,15}$.

Las personas infectadas son contagiosas desde el inicio de la enfermedad, incluso antes de presentar síntomas (lo que dificulta el proceso de aislar enfermos e identificar contactos). Las personas pueden tener una carga viral durante varios días/semanas después de recuperarse, pero no se sabe bien hasta cuándo son infecciosas8. Por eso es un virus con una alta tasa de contagio y una mortalidad estimada del 2 al $4 \%$.

\section{Infección Respiratoria:}

El COVID-I9 causa neumonía severa, con un cuadro agudo sin signos y síntomas prominentes del tracto respiratorio superior, lo que sugiere que las células objetivo del virus están ubicadas en la vía aérea inferior, a nivel alveolar ${ }^{15}$. Este escenario de rápida progresión a un cuadro de insuficiencia respiratoria aguda, ocurre en aproximadamente el $19 \%$ de los casos, requiriendo oxigenoterapia en $14 \%$ y soporte ventilatorio con manejo clínico en Unidad de Cuidados Intensivos en 5\% de los pacientes. En el restante 8I\% de los pacientes, la enfermedad tiene un comportamiento leve o no complicado 8 .

SARS-CoV-2 es un beta-coronavirus $(\beta \mathrm{CoV})$ con alto potencial de neurotropismo. Al parecer, es por esta coinfección del sistema nervioso central que se genera la insuficiencia respiratoria aguda y se aumenta la mortalidad de los casos con neumonía y Síndrome Respiratorio Agudo Severo o Grave $2^{16}$. Sin embargo, otros autores difieren de esa afirmación y sugieren que la clínica de la falla respiratoria mediada por compromiso central es diferente de la secundaria a compromiso respiratorio solo ${ }^{17}$.

La posibilidad de posteriores complicaciones respiratorias y de fibrosis pulmonar, es factible en los pacientes recuperados de neumonía severa por COVID-I9. Sin embargo, solo el tiempo y los resultados clínicos, descartarán esa posibilidad.

\section{Infección Gastrointestinal:}

La detección de ARN del SARS-CoV-2 y la tinción intracelular de la proteína de la nucleocápside viral, en los epitelios gástrico, duodenal y rectal, demuestra que el SARS-CoV-2 infecta las células epiteliales glandulares gastrointestinales. El ARN viral también se detectó en la mucosa esofágica, sin embargo, la ausencia de tinción de la proteína de la nucleocápside viral, indica la baja infección viral en esta mucosa ${ }^{18}$.

Una vez infectado por el virus, aumenta la permeabilidad de la pared gastrointestinal a los patógenos extraños, causando malabsorción en los enterocitos invadidos, lo que produce sínto- 
mas entéricos como diarrea; en teoría esto indica que el sistema digestivo podría ser vulnerable a la infección por COVID-I9 y explica la posibilidad de manifestaciones digestivas asociadas. Algunas series reportan la presencia de síntomas como náuseas y diarrea, sin evidencia de componente respiratorio concomitante, en hasta un $50 \%$ de pacientes, en los cuales posteriormente se confirmó la infección por COVID-I9 ${ }^{19}$.

Se tiene evidencia de hepatotoxicidad asociada al SARS, al parecer secundaria a hepatitis viral o como efecto adverso asociado con toxicidad farmacológica, por las dosis altas de medicamentos antivirales, antibióticos y esteroides, así como probable hiperreactividad del sistema inmune. Sin embargo, en COVID-I9 se ha encontrado importante expresión de ACE2 en colangiocitos $(59.7 \%$ de las células) en lugar de hepatocitos (2.6\% de las células), sugiriendo que podría generar daño de los conductos biliares intrahepáticos ${ }^{20}$.

Adicionalmente, el receptor de enzima convertidora de angiotensina 2 , se expresa ampliamente en las células epiteliales de la mucosa de la cavidad oral ${ }^{21}$. Esta evidencia podría explicar el mecanismo patogénico subyacente de los trastornos olfativos (anosmia) y del gusto (disgeusia), que parecen ser síntomas sensibles y de inicial aparición en pacientes con infección por SARS$\mathrm{CoV}-2^{22}$.

Lo anteriormente expuesto, resalta la importancia de implementar medidas sanitarias de alto impacto a nivel de salud pública, como el distanciamiento social, el lavado de manos y el uso de elementos de protección personal (EPP) para el personal de salud en contacto con los pacientes.

De acuerdo con el CDC (Centers for Disease Control and Prevención) y la OSHA (Occupational Safety and Health Administration), los EPP corresponden a la ropa o equipo especializado utilizado por los empleados para la protección contra materiales infecciosos, que deben ser utilizados de acuerdo con el escenario de exposición. En términos generales, para la atención, prevención y manejo de la infección por SARS-CoV-2/COVID-I9 se consideran los siguientes EPP:
- Guantes.

- Mascarilla quirúrgica.

- Respirador N95, FFP2 o equivalente.

- Protector ocular, careta, monogafa.

- Bata de protección.

- Bata antifluido/Delantal impermeable

- Gorro

- Botas/Zapatos, polainas

\section{Hechos relevantes al realizar procedimientos endoscópicos}

- La endoscopia digestiva produce aerosoles ${ }^{23}$

- La carga viral de SARS-CoV-2 en la saliva faríngea es similar a la de las heces, donde puede persistir dos días más tras negativizarse en muestras respiratorias ${ }^{24}$

- Hasta el 62\% de los contagios por SARS$\mathrm{CoV}-2$ ocurren en fase presintomática ${ }^{25}$

- Mas del 40\% de las infecciones por SARS$\mathrm{CoV}-2$ pueden transmitirse antes de que el caso índice se vuelva sintomático ${ }^{26}$. Dada esta alta tasa de transmisión de infección de individuos pre-sintomáticos, todos los pacientes sometidos a endoscopia digestiva en un área de propagación comunitaria deben considerarse de "alto riesgo" 27

- Todos los procedimientos endoscópicos deben considerarse procedimientos generadores de aerosol (PGA) debido a la posibilidad de regurgitación y tos durante la endoscopia superior y el paso de flatos durante la colonoscopia ${ }^{28}$. Además, todos los procedimientos endoscópicos corren el riesgo de generar aerosoles y microgotas por el diseño, función y posibles fugas de los equipos, válvulas y puertos durante la insuflación y la succión ${ }^{27}$.

- Durante la inserción o salida de accesorios del canal de trabajo del endoscopio, el ajuste de succión e insuflación, retiro de pinza de biopsia y manipulación para extraer el ma- 
terial biológico para patología, y durante el procedimiento de pre-limpieza del equipo, al terminar la endoscopia, pueden generarse movilización de secreciones, aerosoles y microgotas contaminantes ${ }^{23}$.

- De igual manera, todas las superficies de la unidad, almohadas, sabanas, cobijas, carros de transporte, elementos de monitorización, están en riesgo de contaminación y se debe garantizar su limpieza, cambio y adecuada desinfección ${ }^{25,27}$.

- La sala de Endoscopia debe limpiarse y desinfectarse a fondo (limpieza terminal), entre procedimientos. La mayoría de los agentes de limpieza estándar matan este virus ${ }^{28}$.

- El procedimiento de reprocesamiento estándar del endoscopio es suficiente para matar el virus SARS-CoV-2 y debe realizarse de acuerdo con las instrucciones de uso del fabricante de cada equipo ${ }^{28}$.

- Durante una pandemia, todas las personas son consideradas potencialmente contaminantes y deben tratarse como tal. De igual manera, el equipo médico y de atención de salud en el área de endoscopia, debe utilizar todos los elementos de protección personal, como si se tratara de un caso sospechoso.

- Así mismo, existe riesgo de infección COVID-I9 en los pacientes sanos que ingresan a los centros médicos y por lo tanto, durante esta pandemia el paciente debe aceptar esa posibilidad con la firma del consentimiento informado donde se es explícito al respecto ${ }^{29}$.

\section{Recomendaciones para la realizacion de endoscopias}

Dado lo anterior, las recomendaciones sugeridas para la realización de procedimientos endoscópicos son:

I. Los procedimientos de Endoscopia, en estos tiempos de pandemia, se deben limitar a la realización de procedimientos de urgencias e impostergables (como sangrado activo, cuerpo extraño, vólvulo y colangitis) ${ }^{30,31}$.
2. El cuidado de pacientes con cáncer (diagnóstico inicial, estadificación, o paliación por obstrucción), debe individualizarse, debido a que algunos de esos casos pueden considerarse urgencia. Su realización debe definirse idealmente en el contexto de una junta multidisciplinaria ${ }^{23}$.

3. Abstenerse de realizar endoscopias diagnósticas o terapéuticas no urgentes. Si todavía siguieran programadas, deben aplazarse de inmediato. Es necesario mantener la independencia de criterio frente a posibles presiones cuando instancias jerárquicas superiores pretendan actuaciones, que en conciencia y a la luz de los datos científicos disponibles, se consideren perjudiciales ${ }^{32}$.

4. Pacientes que estén en periodo libre de enfermedad de cáncer, sin enfermedad activa, asintomáticos, que requieran estudios endoscópicos de seguimiento, deben programarse después del control de la pandemia, cuando se normalice la atención en los centros médicos ${ }^{33}$.

5. Pacientes asintomáticos que requieran estudios endoscópicos de tamizaje (mayores de 50 años, historia familiar de cáncer o pólipos, etc.), deben programarse después del control de la pandemia, cuando se normalice la atención en los centros médicos ${ }^{33}$.

6. Todos los miembros del equipo de Endoscopia deben usar respiradores N95 (o dispositivos con tasas de filtración equivalentes o más altas), durante la realización de todos los procedimientos gastrointestinales en pacientes con infección conocida por SARS-CoV-2 y en aquellos con alto riesgo de exposición. Dada la alta tasa de transmisión de la infección por individuos pre-sintomáticos, todos los pacientes sometidos a endoscopia digestiva en un área de propagación comunitaria deben considerarse de "alto riesgo" ${ }^{27}$.

7. En los trabajadores de la salud que realizan procedimientos en el tracto gastrointestinal, independientemente del estado de 
COVID-I9, se recomienda el uso de doble guante como parte del equipo de protección personal adecuado ${ }^{34}$.

8. Debe hacerse educación y reentrenamiento al talento humano de la Unidad de Endoscopia, sobre el procedimiento adecuado para ponerse y quitarse los EPP ${ }^{28}$.

9. Es obligatorio lavarse o desinfectarse las manos antes de ponerse y después de quitarse los EPP ${ }^{28}$.

IO. Los procedimientos con mayor riesgo de generación de aerosoles idealmente deben realizarse en una sala con presión negativa, si se cuenta con ella ${ }^{28}$.

II. Para procedimientos que requieren anestesia endotraqueal:

- La intubación y la extubación deben realizarse idealmente en una sala con presión negativa ${ }^{28}$.

- El personal de endoscopia no debe estar en la habitación durante la intubación y extubación ${ }^{28}$.

\section{Discusión}

Es importante reconocer la importancia del uso regular, constante y reglado de los elementos de protección personal en el trabajo. Se cree que todo el talento humano en salud conoce la premisa de bioseguridad de: "primero nuestra protección y estaremos listos para atender de la mejor manera a los pacientes". Sin embargo, una realidad que ha puesto en evidencia esta situación de pandemia, es que no estaba cumpliéndose esa norma en muchas instituciones. Y la responsabilidad de ello está compartida entre empleadores y trabajadores, evidenciando que el comportamiento individual es fundamental para la implementación y adopción de medidas sanitarias. Una lección importante de esta experiencia es: "La bioseguridad va primero, es responsabilidad individual y una conducta de autocuidado que debe priorizarse".
Adicionalmente, en este momento no tenemos disponible un tratamiento específico, una vacuna, anticuerpos o un suero convaleciente efectivo, para tratar a los pacientes. Cada día aparecen nuevas opciones de manejo, que deben individualizarse según el caso y condiciones particulares de cada paciente.

Hay desenlaces inesperados y no tenemos todavía toda la información que quisiéramos para combatir el virus y dar un manejo eficaz a nuestra población, exento de complicaciones. Existe cierto grado de incertidumbre, que se irá esclareciendo a medida que se obtengan más datos y se realicen más investigaciones, por lo que es probable que las recomendaciones cambien sobre la base de los nuevos conocimientos que se obtengan. Una mayor certeza permitirá una formulación de políticas y una toma de decisiones individuales mejor informadas ${ }^{35,36}$.

El panorama actual está exigiendo al talento humano en salud, actuar con sabiduría, informarse de fuentes confiables, trabajar en equipo, apoyarse en la experiencia y el sentido común, compartiendo ideas con su grupo de trabajo para generar soluciones y respuestas, siendo vulnerables y pidiendo apoyo si se requiere, para ser fuentes de una atención en salud de calidad, humana, oportuna y eficaz, al servicio de la sociedad.

La esperanza debe estar viva, los cambios son positivos y el mundo estaba pidiendo un respiro de muchas cosas, situaciones y relaciones, que no estaban funcionando y que ahora, como sociedad, tenemos la oportunidad de transformar en una bonita realidad de solidaridad, humildad, paciencia, crecimiento, aprendizaje y sanación.

En conclusión, no todo depende del sector salud y debemos aceptarlo, ya que se presentan desenlaces que no se pueden prever ni controlar. Sin embargo, existen variables modificables, como son las conductas de autocuidado, bioseguridad individual y del grupo de trabajo, cumplimiento de las medidas de distanciamiento social, lavado de manos y demás recomendaciones generadas por las sociedades científicas y autoridades sanitarias, que son responsabilidad 
del personal en salud y comunidad en general, el acatar y cumplir. El talento humano en salud con su compromiso ético y resilencia, es fuente, para una sociedad que requiere que la educación, se enseñe con el ejemplo.

\section{Cumplimiento de normas éticas}

Consentimiento informado: Esta publicación es una revisión de la literatura, y como tal no hay necesidad de un consentimiento informado ni de aprobación del Comité de Ética Institucional.

Declaración de conflicto de intereses: La autora declaró que no tiene conflicto de intereses.

Fuentes de financiación: Recursos propios de la autora.

\section{Referencias}

I. World health organization (WHO). Novel Coronavirus (20I9-nCoV) Situation Report - 7 [cited 28 January]. Disponible en: https://www.who.int/ docs/default-source/Coronaviruse/situationreports/20200I27-sitrep-7-2019-ncov.pdf?sfvrs$\mathrm{n}=98 \mathrm{ef} 79 \mathrm{f} 5 \_2$

2. Instituto Nacional de Salud, Colombia. Anexo. Instructivo para la vigilancia en salud pública intensificada de infección respiratoria aguda asociada al nuevo coronavirus 2019 (COVID-I9). Fecha de actualización: sábado 04 de abril 2020 Versión: 9. Disponible en: https://www.ins.gov.co/Noticias/Coronavirus/Anexo_\%2oInstructivo\%2oVigilancia\%2oCOVID\%20 v9\%2004042020.pdf

3. Millan-Oñate J, Rodríguez-Morales AJ, Camacho-Moreno G, Mendoza-Ramírez H, Rodríguez-Sabogal IA, Álvarez-Moreno C. A new emerging zoonotic virus of concern: the 2019 novel Coronavirus (COVID-I9). Infectio 2020;24.

4. Chen N, Zhou M, Dong X, Qu J, Gong F, Han Y, et al. Epidemiological and clinical characteristics of 99 cases of 2019 novel coronavirus pneumonia in Wuhan, China: a descriptive study. The Lancet. 2020 http:// doi:IO.IOI6/SoI40- 6736(20)302II-7.

5. Huang C, Wang Y, Li X, Ren L, Zhao J, Hu Y, et al. Clinical features of patients infected with 2019 novel coronavirus in Wuhan, China. The Lancet. 2020;395:497-506.

6. Li Q, Guan X, Wu P, Wang X, Zhou L, Tong Y, et al. Early transmission dynamics in Wuhan, China, of Novel Coronavirus-Infected Pneumonia. N Engl J Med. 2020.

7. Munster VJ, Koopmans M, van Doremalen N, van Riel D, de Wit E. A novel coronavirus emerging in China
- Key questions for impact assessment. N Engl J Med. 2020;382:692-4.

8. Consenso colombiano de atención, diagnóstico y manejo de la infección por SARS-COV-2/COVID-I9 en establecimientos de atención de la salud. Recomendaciones basadas en consenso de expertos e informadas en la evidencia Grupo ACIN- IETS de Consenso Colombiano para recomendaciones de atención COVIDi9. http://dx.doi.org/IO.22354/in.v24i3.85I

9. Centers for Disease Control and Prevention (CDC). About Novel Coronavirus (2019-nCoV) | CDC Symptoms 2020 [cited 28 January 2020]. Disponible en: https://www.cdc.gov/Coronavirus/20I9-ncov/about/ index.html

Io. WHO Director-General's opening remarks at the media briefing on COVID-I9 - II March 2020 [cited I7 March 2020]. Disponible en: https://www.who.int/ $\mathrm{dg} /$ speeches/detail/who-director-general-s-opening-remarks-at-the-media-briefing-on-covid-I9--II-march-2020

II. Ministerio de Salud y Protección Social. Boletín de prensa No III de 2020. [cited o4 April 2020]. Disponible en: https:/www.minsalud.gov.co/Paginas/Colombia-entra-en-fase-de-mitigacion-de-la-COVID-I9. aspx

I2. Hamming I, Timens W, Bulthuis ML, Lely AT, Navis $\mathrm{G}$, van Goor H.Tissue distribution of ACE2 protein, the functional receptor for SARS coronavirus. A first step in understanding SARS pathogenesis. J Pathol. 2004;203:63I-7.

I3. Wan Y, Shang J, Graham R, Baric RS, Li F. Receptor recognition by novel coronavirus from Wuhan: An analysis based on decadelong structural studies of SARS. J Virology 2020; published online Jan 29. https:doi.org/IO.II28/JVI.ooI27-20.

I4. Michael M.C.Lai, David Cavanagh. The molecular biology of coronaviruses. Advances in Virus Research. 1997;48:I-IOo. https://doi.org/IO.IOI6/Soo653527(08)60286-9

15. Paul S. Masters. The Molecular Biology of Coronaviruses. Advances in Virus Research. 2006;66:193-292. https://doi.org/Io.IoI6/Soo65-3527(06)66005-3

I6. Li Y-C, Bai W-Z, Hashikawa T. The neuroinvasive potential of SARS-CoV2 may play a role in the respiratory failure of COVID-I9 patients. J Med Virol.2020;I4. https://doi.org/IO.IOO2/jmv.25728

I7. Turtle L. Respiratory failure alone does not suggest central nervous system invasion by SARS-CoV-2. J Med Virol. 2020 Apr 4. https://doi.org/IO.IOO2/ jmv.25828

I8. Xiao F, Tang M, Zheng X, Liu Y, Li X, Shan H. Evidence for gastrointestinal infection of SARS-CoV-2. Gastro- 
enterology, article in press. https://doi.org/IO.IO53/j. gastro.2020.02.055

I9. Gu J, Han B, Wang J. COVID-I9: Gastrointestinal Manifestations and Potential Fecal-Oral Transmission. Gastroenterology, article in press. https://doi. org/IO.IO53/j.gastro.2020.02.054

20. Chai XQ, Hu LF, Zhang Y, Han W, Lu Z, Keet A, et al. Specific ACE2 expression in cholangiocytes may cause liver damage after 2019-nCoV infection. Preprint. Posted online February 03, 2020. bioRxiv 931766. https:// doi.org/IO.IIOI/2O2O.02.03.93I766

2I. Xu H, Zhong L, Deng J, Peng J, Dan H, Zeng X, et al. High expression of ACE2 receptor of 20I9- $\mathrm{nCoV}$ on the epithelial cells of oral mucosa. Int J Oral Sci. 2020;12:8. https://doi.org/IO.IO38/s4I368-020-0074-X.

22. Giacomelli A, Pezzati L, Conti F, Bernacchia D, Siano M, Oreni L, et al. Self-reported olfactory and taste disorders in SARS-CoV-2 patients: a cross-sectional study. Clinical Infectious Diseases, article in press. https://doi.org/IO.IO93/cid/ciaa330

23. Soetikno R, Teoh AYB, Kaltenbach T, Lau JY, Asokkumar R, Cabral-Prodigalidad P, et al. Considerations in performing endoscopy during the COVID-I9 pandemic. Gastrointest Endosc 2020 Mar 19. https://doi. org/IO.IOI6/j.gie.2020.03.3758.

24. Coronavirus can persist in stool after its clearance in respiratory tract. Faculty of Medicine, CUHK Press Release. Accessed 2020 Mar 2I. Disponible en: https://www.med.cuhk.edu.hk/press-releases/ cuhk-finds-that-the-coronavirus-canpersist-instool-after-its-clearance-in-respiratory-tractwill-conduct-stool-test-forpeople-in-quarantinecamps-for-early-identification

25. Ganyani T, Kremer C, Chen D, Torneri A, Faes C, Wallinga J, et al. Estimating the generation interval for COVID-I9 based on symptom onset data. medRxiv 2020 Mar 8: 2020.03.05.20031815 https://doi. org/IO.IIOI/2020.03.05.2003I8I5

26. He X, Lau EHY, Wu P, DengX, Wang J, Hao X, et al. Temporal dynamics in viral shedding and transmissibility of COVID-I9. medRxiv 2020; March I5. https:// doi.org/IO.IIOI/2020.03.I5.20036707

27. American Gastroenterological Association - AGA. Joint gastroenterology society message: COVID-I9 use of personal protective equipment in GI endoscopy, April or 2020, Disponible en: https://webfiles.gi.org/ links/media/JOINT_GI_SOCIETY_MESSAGE_ PPE_FINAL_040I2020.pdf
28. World Endoscopy Organization - WEO recommendations on digestive endoscopy and the COVID-I9 pandemic. 24.03.2020. Disponible en: http://www. worldendo.org/2020/03/24/weo-advice-on-digestive-endoscopy-and-the-covid-r9-pandemic/

29. Otero R, Gómez-Zuleta MA, Angel-Arango LA, Ruíz-Morales OF, Marulanda-Fernández H, Riveros J, et al. Procedimientos endoscópicos y pandemia COVIDig. Consideraciones básicas. Rev Colomb Gastroenterol. 2020;35;65-75. https://doi.org/IO.225I6/25007440.526

30. Sociedad Colombiana de Endoscopia Digestiva. Comunicado No. 3 a la Opinión Publica: 03 de abril de 2020.

3I. Zhang Y, Zhang X, Liu L, Wang H, Zhao Q. Suggestions of Infection Prevention and Control in Digestive Endoscopy During Current 2019-nCoV Pneumonia Outbreak in Wuhan, Hubei Province, China. [acceso 2020 Mar 23]; Disponible en: http://www. worldendo.org/wp-content/uploads/2020/02/ Suggestions-of-Infection-Prevention-and-Control-in-Digestive-Endoscopy-During-Current-20I9-nCoV-Pneumonia-Outbreak-in-Wuhan-Hubei-Province-China.pdf.

32. Sociedad Española de Endoscopia Digestiva - SEED. Comunicado 2I/03/2020 | Actualizado 22/03/2020 a las I4:38 horas.

33. American Society of Clinical Oncology (ASCO). COVID-I9 Patient Care information. Disponible en: https:/www.asco.org/asco-coronavirus-information/care-individuals-cancer-during-covid-I9

34. American Gastroenterological Association - AGA. AGA issues formal recommendations for PPE during gastrointestinal Procedures. March 3I 2020, Disponible en: https://www.gastro.org/news/aga-issues-formal-recommendations-for-ppe-during-gastrointestinal-procedures

35. Organización Mundial de la salud. Orientación ética sobre cuestiones planteadas por la pandemia del nuevo coronavirus (COVID-I9), I8 de marzo 2020. Disponible en: https://www.paho.org/es/documentos/orientacion-etica-sobre-cuestiones-planteadas-por-pandemia-nuevo-coronavirus-covid-I9

36. Organización Panamericana de la Salud. Pautas de la OMS sobre la ética en la vigilancia de la salud pública. Washington DC: OPS; 20I7. Disponible en: https:// iris.paho.org/bitstream/handle/I0665.2/34499/97 89275319840-spa.pdf? sequence $=6 \&$ is Allowed $=\mathrm{y}$ 